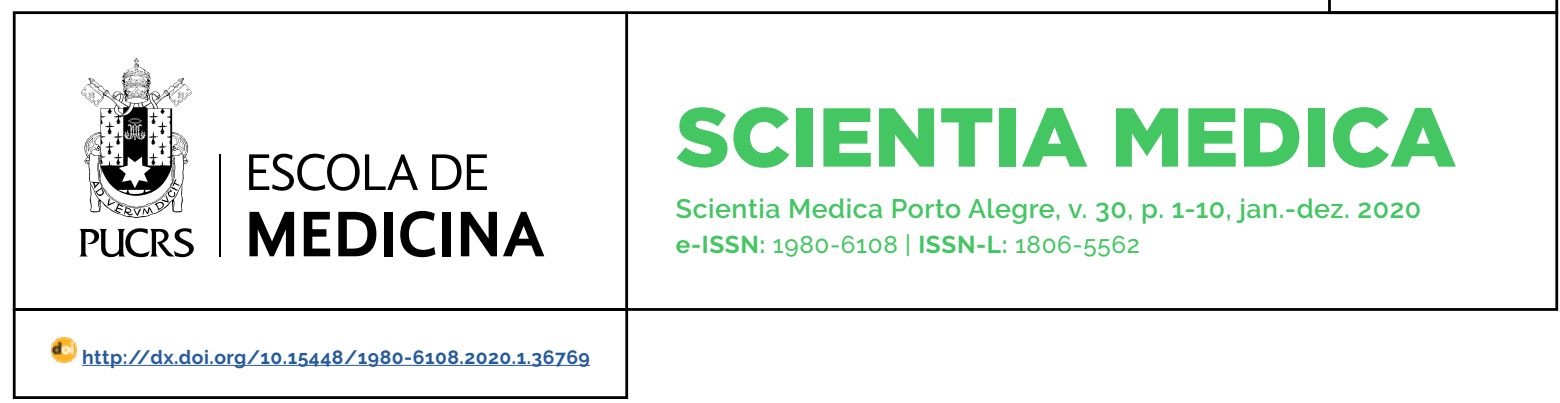

ARTIGO ORIGINAL

\title{
Determinantes da relação entre percepção do funcionamento do sistema urinário atrapalhar a vida e a qualidade de vida de longevos
}

\author{
Determinants of the relationship between perception of urinary system function annoys \\ the life and quality of life in oldest-old
}

\author{
Luisa Braga Jorge ${ }^{1}$ \\ orcid.org/0000-0003-2695-8340 \\ lbragajorge@hotmail.com
}

\section{Josemara de Paulo}

Rocha $^{1}$

orcid.org/0000-0003-2809-5737

josemara.rocha@hotmail.com

\section{Ângelo José Gonçalves \\ Bós ${ }^{1}$}

orcid.org/0000-0003-4901-3155

angelo.bos@pucrs.br

\section{Rodolfo Herberto \\ Schneider ${ }^{1}$}

orcid.org/0000-0001-6946-8056

rodolfo.schneider@pucrs.br

Recebido em: 6 jan. 2020. Aprovado em: 4 abr. 2020. Publicado em: 24 jul. 2020

\section{(c) (i)}

Artigo está licenciado sob forma de uma licença Creative Commons Atribuição 4.0 Internacional.

\section{Resumo}

Objetivo: este estudo buscou avaliar a relação entre a percepção do funcionamento do sistema urinário atrapalhar a vida de modo geral com a análise do questionário Kings Health Questionnaire (KHQ) em longevos.

Métodos: estudo transversal e analítico. Foram investigadas caracteristicas sociodemográficas e clínicas, bem como, o impacto da incontinência urinária (IU) na Qualidade de vida (QV) pelo instrumento KHQ. Frequências, médias e desvio-padrão, testes de qui-quadrado, t de student e regressões logísticas foram calculadas, aceitando como significativos valores de $p<0,05$.

Resultados: participaram 82 longevos, 68\% mulheres, idade média de 92,3 $\pm 2,7$ anos, $71 \%$ incontinentes e $43 \%$ referindo que a funcionalidade pélvica atrapalhava a vida. Individuos incontinentes relataram mais frequentemente a queixa investigada. Os domínios, impacto da IU, emoções, limitação físico-social, limitação no desempenho de tarefas do $\mathrm{KHQ}$ e seu escore total foram relacionados com a queixa. Individuos que relataram limitação de tarefas fora de casa, em viagens, vontade forte de urinar e dificil de controlar, sentimento de depressão e ansiedade ou nervosismo tinham aumento significativo na chance de relatar que a funcionalidade pélvica atrapalhava a vida.

Conclusão: o KHQ foi relacionado com a chance de relato de que o funcionamento do sistema urinário atrapalhava a vida. Mesmo assim, boa parte dos incontinentes não relatava que a condição atrapalhava a vida. Por isso, os resultados demonstram que mesmo na ausência de afirmação positiva de ausência de IU, o funcionamento do sistema urinário pode comprometer a QV. Os achados sugerem que, ou os participantes realmente tinham IU e não a percebiam como tal, ou que é necessário investigar outras questões como frequência miccional, por exemplo. O uso do KHQ permitiu identificar que mesmo na ausência de relato de IU o funcionamento do sistema urinário pode interferir na QV de longevos.

Palavras-chave: idoso de 80 anos ou mais, sistema urinário, sintomas do trato urinário inferior, incontinência urinária, qualidade de vida.

\section{Abstract}

Aims: this study aimed to evaluate the relationship between the perception that urinary system function annoys the life in general with the analysis of the Kings Health Questionnaire (KHQ) in old age.

Methods: cross-sectional and analytical study. Sociodemographic and clinical characteristics were investigated, as well as the impact of urinary incontinence (UI) on Quality of Life (QOL) by the KHQ instrument. Frequencies, means and standard deviation and chi-square, student t tests and logistic regressions were calculated, accepting as significant $p$ values $<0.05$.

Results: 82 elderly, $68 \%$ women, mean age $92.3 \pm 2.7$ years, $71 \%$ incontinent and $43 \%$ reporting that the pelvic functionality disrupted life. Incontinent individuals most frequently reported the investigated complaint. The domains, UI impact, emotions, physical and social limitation, KHQ task performance limitation and its 
total score were related to the complaint. Individuals who reported limitation of tasks away from home, travel, strong desire to urinate and difficult to control, feelings of depression and anxiety or nervousness had a significant increase in the chance of reporting that pelvic functionality was disruptive to life.

Conclusion: $\mathrm{KHQ}$ was related to the chance of reporting that the urinary system function was disruptive to life. Even so, most incontinents did not report that the condition disrupted life. Therefore, the results show that even in the absence of a positive statement of absence of $\mathrm{UI}$, the urinary system function can compromise QOL. The findings suggest that either participants actually had UI and did not perceive $\mathrm{UI}$ as such, or that further questions such as voiding frequency need to be investigated, for example. The use of $\mathrm{KHQ}$ allowed us to identify that even in the absence of $\mathrm{UI}$ report, the urinary system function can interfere with the QoL of the oldest old.

Keywords: aged 80 years or older, urinary tract, lower urinary tract symptoms, urinary incontinence, quality of life.

ABREVIATURAS: ABVD, atividades básicas de vida diária; AIVD, atividades instrumentais de vida diária; AMPAL, Atenção Multiprofissional ao Longevo; IU, incontinência urinaria; QV, qualidade de vida.

\section{Introdução}

A Organização das Nações Unidas estima que nos próximos 43 anos o número de pessoas com mais de 60 anos de idade será três vezes maior do que atualmente. Destaca-se no último censo o aumento importante e crescente das pessoas com 80 anos ou mais, também chamadas de longevos (1).

No Brasil, ainda são poucas as pesquisas que fazem abordagem especifica a essa faixa de idade. Devido a isso, justificam-se estudos que buscam ampliar o conhecimento sobre as condições de vida e de saúde dos longevos, pois ao evidenciarmos as reais necessidades, é possivel traçar um planejamento sobre prevenção e tomada de decisão na busca por mais qualidade de vida (QV) (2).

A longevidade pode vir acompanhada de alguns prejuizos funcionais do corpo e da mente. O que pode predispor a eventos incapacitantes e que geram uma perda muito grande da QV (3). Entre esses eventos, a diminuição do funcionamento da bexiga não é o único fator que pode levar a sintomas urinários no envelhecimento. $\mathrm{O}$ enfraquecimento dos músculos pélvicos, que também acontece na senescência, pode, da mesma forma, contribuir para disfunções urinárias, como por exemplo, levantar-se muitas vezes durante a noite para ir ao banheiro, aumento da frequência miccional e perda de urina (4).

Apesar da incontinência urinária (IU) não ser uma condição assustadora em termos de gravidade, a literatura descreve que o nivel de QV baixa drasticamente, podendo levar ao aparecimento de quadros depressivos, isolamento social, constrangimento, diminuição da função sexual, qualidade do sono/repouso e limitações de atividades diárias e ocupacionais (5). Além disso, é um marcador que pode aumentar a chance de institucionalização, podendo ter consequências físicas como lesões de pele, dermatite amoniacal, propensão a úlceras de decúbito, infecção urinária e quedas (6).

Através da identificação da prevalência e dos fatores associados a IU podem ser traçadas e planejadas medidas de prevenção e tratamento que podem reduzir os sintomas e os custos da IU e melhorem a QV dos longevos (7). Já que essa doença, considerada uma das grandes sindromes geriátricas, está relacionada com menor frequência do sair de casa (8), quedas (9) e mortalidade (10) na longevidade. Sendo assim, o objetivo desta pesquisa é avaliar a relação da funcionalidade pélvica com a qualidade de vida de idosos

\section{Métodos}

Trata-se de um estudo transversal, observacional, analítico de caráter quantitativo. Os participantes foram selecionados no ano de 2016 e integraram um estudo longitudinal, denominado Atenção Multiprofissional ao Longevo (AMPAL), da Pontifícia Universidade Católica do Rio Grande do Sul (PUCRS). O AMPAL tem acompanhado individuos acima de 87 anos, principalmente nonagenários, residentes em domicilios de Porto Alegre, RS, Brasil, independentemente do estado funcional, buscando traçar um perfil dessa população e realizando um acompanhamento, através de amostra de conglomerados representativos das regiões dos orçamentos participativos da cidade de Porto Alegre. Após cálculo para obtenção do tamanho amostral, foi sugerido o acompanhamento de $10 \%$ dos nonagenários de Porto Alegre, que seria em torno de 480 sujeitos, 
já que a cidade, de acordo com o censo do Instituto Brasileiro de Geografia e Estatística de 2010, apontava para um total de quase 5 mil pessoas nesse grupo etário. O estudo não incluiu longevos institucionalizados ou hospitalizados (11).

Para a presente pesquisa foram selecionados homens e mulheres com mais de 85 anos participantes do AMPAL, capazes de responder com clareza às questões do estudo e que tinham respondido à questão "o quanto a urina ou bexiga atrapalham a sua vida?" no questionário de avaliação do AMPAL. Os longevos que não se ajustavam a essas condições e apresentavam informações incompletas no banco de dados não foram incluídos no estudo.

Aleatoriamente, os possiveis sujeitos da pesquisa (longevos do AMPAL) eram contactados por telefone e as avaliações, realizadas nos domicilios deles. A avaliação incluía um questionário multidimensional, onde constavam várias perguntas gerais de saúde, adaptadas pelo AMPAL a partir do caderno número 19 de atenção à saúde da pessoa idosa do ministério da saúde brasileiro (12). Nesse caderno consta a pergunta "O/A Sr/a, às vezes, perde urina ou fica molhado/a? Se sim, pergunte: Quantas vezes? Isso provoca algum incomodo ou embaraço?" (p. 48). Foram investigados fatores sociodemográficos e clínicos e o impacto da percepção do individuo sobre o funcionamento do sistema urinário sobre qualidade de vida através do Kings Health Questionnaire (KHQ) e uma modificação na questão do caderno 19, pois foi questionada a percepção do funcionamento do sistema urinário em indivíduos sem queixa de perda urinária. Essa adaptação foi inspirada no estudo de Zanella (13), que em sua tese de Doutorado, utilizando o KHQ, observou que um número considerável de idosas responderam que a bexiga ou a urina atrapalhava a vida mesmo na ausência de IU. Relatou também, a existência de baixa QV relacionada a essa questão, em alguns casos, independente da presença de IU.

A possibilidade de a rotina do sistema urinário atrapalhar a vida dos participantes foi investigada através da questão "a urina ou a bexiga atrapalha a sua vida ou não". Foi considerado atrapalhar a bexiga as respostas "raramente ou pouco", "às vezes ou mais ou menos" e "frequentemente ou muito" e que não atrapalhava, as atribuições, "nada" ou "quase nada". Esses dois grupos foram assim categorizados para fins de análise estatística, independente de apresentarem ou não incontinência urinária. Já a continência foi investigada através da questão "O/A Sr./a às vezes perde urina ou fica molhado/a?".

$\mathrm{O} \mathrm{KHQ}$ foi validado para ser utilizado no Brasil e é composto por 30 questões, divididas em nove domínios: percepção geral de saúde, impacto da incontinência urinária, limitações ao desempenho de tarefas, limitações físicas, limitações sociais, relacionamento pessoal, emoções e sono/disposição e medidas de gravidade. Além destes domínios, existe a escala de sintomas composta por frequência urinária, noctúria, urgência miccional, incontinência urinária de esforço, enurese noturna, incontinência no intercurso sexual, infecções urinárias e dor na bexiga (1). Embora esse questionário tenha sido inicialmente desenvolvido para sujeitos com incontinência, outro estudo já utilizou para grupos continentes (6).

Para essas perguntas existem quatro opções de respostas ("nem um pouco, um pouco, moderadamente, muito" ou "nunca, às vezes, frequentemente, o tempo todo"), exceção feita ao domínio percepção geral de saúde com cinco opções de respostas ("muito boa, boa, regular, ruim, muito ruim") e o domínio relações pessoais ("não aplicável, nem um pouco, um pouco, moderadamente e muito"). Os valores são calculados por domínios individuais somados e avaliados através de uma fórmula matemática, obtendo-se, assim, o escore de QV, que varia de o a 100, considerando-se que quanto maior o número obtido, pior a QV (14)

Primeiramente foi testada a relação entre a queixa de que o funcionamento do sistema urinário atrapalhava a vida com as variáveis sociodemográficas e a continência através dos testes estatísticos de qui-quadrado e teste t de student. Quando os dados não tinham distribuição paramétrica foi optado pelo teste exato de Fisher e Kruskal Wallis em substituição aos testes mencionados. Em seguida, através do teste $\mathrm{t}$ de student foram 
comparadas as médias obtidas em cada um dos dominios do $\mathrm{KHQ}$ com as categorias "atrapalha" e "não atrapalha" respondidas na questão sobre a relação entre a percepção do funcionamento do sistema urinário e a vida dos participantes. Finalmente, foram elaborados modelos de regressão logística univariada para estudar qual era a chance dos individuos que relataram limitações nos domínios do $\mathrm{KHQ}$ também relatarem que o funcionamento do sistema urinário atrapalhava a vida. Para cada modelo univariado, em seguida era realizado um novo modelo ajustado pela incontinência urinária para avaliar o comportamento da mesma sobre o efeito de cada questão do $\mathrm{KHQ}$ sobre a chance de relato de o funcionamento do sistema urinário atrapalhar a vida. Para todos os testes se aceitou um intervalo de confiança de 95\% como significativo e o Epi Info 7.2.2.6 foi o software utilizado para as análises.

Esse projeto de pesquisa foi aprovado pela Comissão Científica do Programa de Pós-Graduação em Gerontologia Biomédica da PUCRS e do Comitê de Ética em Pesquisa da PUCRS sob número CAAE: 59664716.50000.5336.

\section{Resultados}

A amostra foi representada por 82 longevos, idade média de 92,3 $\pm 2,7$ anos, principalmente viúvos (67\%), baixa escolaridade (38\% sem escolaridade) e sexo feminino (68\%) (Tabela 1). Entre os longevos que relataram que o funcionamento do sistema urinário atrapalhava a vida, identificou-se maior proporção de viúvos (74\%), baixa escolaridade (43\% sem escolaridade) e mulheres (77\%) (Tabela 1), porém as diferenças não foram estatisticamente significativas. Individuos incontinentes relataram mais frequentemente que o funcionamento do sistema urinário atrapalhava a vida. Porém, é interessante observar que entre os longevos que não relatavam esse problema, praticamente não havia diferença no percentual de continente e incontinentes (Tabela 1).

TABELA 1 -. Percepção do funcionamento do sistema urinário atrapalhar a vida e a relação com fatores sociodemográficos e continência

\begin{tabular}{|c|c|c|c|c|}
\hline & Atrapalha & Não atrapalha & Total & $p$ \\
\hline Estado Civil & & & & 0,230 \\
\hline Viúvos, n(\%) & $26(74)$ & $29(62)$ & $55(67)$ & \\
\hline Não viúvos, n(\%) & $9(26)$ & $18(38)$ & $27(33)$ & \\
\hline Nivel de escolaridade & & & & 0,494 \\
\hline Sem escolaridade, n(\%) & 14(43) & $15(31)$ & $31(38)$ & \\
\hline Fundamental, n(\%) & $8(24)$ & $19(41)$ & 27 (33) & \\
\hline Médio, n(\%) & $7(21)$ & $7(15)$ & $14(17)$ & \\
\hline Superior, n(\%) & $4(12)$ & $6(13)$ & 10(12) & \\
\hline Sexo & & & & 0,137 \\
\hline Feminino, $\mathrm{n}(\%)$ & $27(77)$ & $29(62)$ & $56(68)$ & \\
\hline Masculino, n(\%) & $8(23)$ & $18(38)$ & $26(32)$ & \\
\hline Idade (anos), médiaะDP & $92,6 \pm 2,5$ & $92,1 \pm 2,7$ & $92,3 \pm 2,6$ & 0,456 \\
\hline \multicolumn{5}{|l|}{ Continência urinária } \\
\hline Continentes, n(\%) & $1(3)$ & $23(49)$ & $24(29)$ & $<0,001$ \\
\hline Incontinentes, n(\%) & 34 (97) & $24(51)$ & $58(71)$ & \\
\hline
\end{tabular}

Testes estatísticos: qui-quadrado para variáveis categóricas; t de student para a idade. 
Quatro médias dos dominios do KHQ foram relacionadas com o relato de o funcionamento do sistema urinário atrapalhar ou não a vida dos participantes, "impacto da incontinência urinária", "emoções", "limitação físico-social" e "limitação no desempenho de tarefas" ( $p<0,001)$. O escore geral foi pior para aqueles que relatavam que a condição não atrapalhava a vida. Contudo, analisando domínio por domínio, o KHQ, ou seja, o impacto da funcionalidade pélvica urinária sobre a qualidade de vida, foi maior para aqueles que relacionavam comprometimento emocional, limitação fisico-funcional e no desempenho de tarefas ao problema de bexiga (Tabela 2). 0 dominio "impacto da incontinência urinária" do KHQ tinha pior média (mais alta) entre os longevos que relataram que o funcionamento do sistema urinário não atrapalhava a vida $(p<0,001)$.

TABELA 2 - Valores das médias dos escores dos dominios do KHQ entre os participantes com e sem queixa de que o sistema urinário atrapalhava a vida

\begin{tabular}{|c|c|c|c|c|}
\hline Escores & Atrapalha & Não atrapalha & Total & $p$ \\
\hline Medidas de gravidade & $74,8 \pm 7,4$ & $79,0 \pm 13,1$ & $77,2 \pm 11,2$ & 0,092 \\
\hline Limitação nas relações pessoais & $62,4 \pm 33,0$ & $51,4 \pm 33,7$ & $56,1 \pm 33,6$ & 0,147 \\
\hline Sono/Energia & $36,6 \pm 4,1$ & $37,7 \pm 3,4$ & $37,3 \pm 3,7$ & 0,221 \\
\hline Percepção negativa de Saúde & $29,3 \pm 21,4$ & $22,3 \pm 17,4$ & $25,3 \pm 19,4$ & 0,110 \\
\hline Impacto da IU & $20,7 \pm 0,9$ & $22,2 \pm 1,1$ & $21,6 \pm 1,3$ & $<0,001$ \\
\hline Emoções & $34,3 \pm 27,5$ & $7,08 \pm 13,3$ & $18,7 \pm 24,5$ & $<0,001$ \\
\hline Limitação físico-social & $14,2 \pm 2,3$ & $13,0 \pm 0,3$ & $13,5 \pm 1,6$ & $<0,001$ \\
\hline Limitação desempenho tarefas & $12,8 \pm 16,1$ & $2,85 \pm 8,0$ & $7,1 \pm 13,0$ & $<0,001$ \\
\hline Escore Total & $21,3 \pm 0,8$ & $22,7 \pm 0,9$ & $22,13 \pm 1,1$ & $<0,001$ \\
\hline
\end{tabular}

Teste estatístico= t de student. IU, Incontinência urinária; KHQ, Kings Health Questionnaire.

Nos modelos de regressão logística univariados foi possivel identificar que todos os dominios do KHQ estavam associados com a chance de o funcionamento do sistema urinário atrapalhar a vida dos longevos, principalmente quando eles relacionavam o problema na bexiga com limitações de tarefas fora de casa (RC $=13,28 ; 1$ C $95 \%$ $=2,75-64,07)$, em viagens $(R C=21,08 ; 1 C 95 \%=$ 2,56 - 173,15), e ansiedade ou nervosismo (RC = 12,59; IC95\% = 3,99 - 39,69). Interessante que o uso de protetores higiênicos, se sentir mal por esses problemas de urina ou bexiga, sentimento de depressão, vontade forte de urinar e dificil de controlar foram fatores protetores para a queixa de atrapalhar a vida (Tabela 3). Todavia, quando as variáveis foram individualmente ajustadas pelo relato de perda urinária, apenas permaneceram significativamente relacionadas, limitações de tarefas fora de casa, limitação em viagens, vontade forte de urinar e dificil de controlar, sentimento de depressão, e ansiedade ou nervosismo (Tabela 3). A perda urinária incluida nos modelos univariados, fez com que vários domínios e questões do KHQ deixassem de ser significativamente relacionados com a queixa de atrapalhar a vida, limitação em visitar amigos ou parentes, durante a vida social ou familiar, perda de urina quando tem vontade forte de urinar, incontinência urinária de esforço, perda de urina durante o sono, infecção urinária frequente, se sentir mal por esses problemas de urina e bexiga, uso de protetores higiênicos e preocupação em cheirar urina (Tabela 3). 
TABELA 3 - Modelos de regressão logística univariados e ajustados pela presença de incontinência urinária (IU) para a chance de percepção do funcionamento do sistema urinário atrapalhar a vida de acordo com os domínios do $\mathrm{KHQ}$

\begin{tabular}{|c|c|c|c|c|}
\hline \multirow{2}{*}{$\begin{array}{c}\text { Perguntas KHQ (referência: ausência } \\
\text { da limitação ou sintoma) }\end{array}$} & \multicolumn{2}{|c|}{ Modelos univariados } & \multicolumn{2}{|c|}{$\begin{array}{l}\text { Modelos anteriores } \\
\text { ajustados pela IU }\end{array}$} \\
\hline & $\mathrm{RC}\left(\mathrm{IC}_{95 \%)}\right.$ & $p$ & $\mathrm{RC}\left(\mathrm{IC}_{95} \%\right)$ & $p$ \\
\hline Limitação de tarefas fora de casa & $13,28(2,75-64,07)$ & $<0,001$ & $10,78(1,79-64,88)$ & 0,009 \\
\hline Limitação em viagens & $21,08(2,56-173,15)$ & 0,004 & $10,99(1,31-92,29)$ & 0,027 \\
\hline Limitação em visitar amigos ou parentes & $9,51(1,08-83,08)$ & 0,041 & $15,05(0,82-274,8)$ & 0,067 \\
\hline Limitação durante a vida social ou familiar & $2,99(1,04-8,62)$ & 0,042 & $1,21(0,33-4,45)$ & 0,766 \\
\hline Vontade forte de urinar e difícil de controlar & $0,09(0,03-0,26)$ & $<0,001$ & $0,17(0,05-0,52)$ & 0,001 \\
\hline $\begin{array}{l}\text { Perda de urina quando tem vontade forte } \\
\text { de urinar }\end{array}$ & $6,81(2,25-20,61)$ & $<0,001$ & $0,68(0,11-4,06)$ & 0,674 \\
\hline Incontinência urinária de esforço & $3.92(1,54-9,94)$ & 0,004 & $1,36(0,47-3,94)$ & 0,563 \\
\hline Perda de urina durante o sono & $4,29(1,21-15,15)$ & 0,023 & $2,76(0,70-10,94)$ & 0,146 \\
\hline Infecção urinária frequente & $3.72(104-13,30)$ & 0,043 & $3,41(0,75-15,35)$ & 0,110 \\
\hline Sentimento de depressão & $0,11(0,04-0,31)$ & $<0,001$ & $0,22(0,07-0,68)$ & 0,008 \\
\hline Ansiedade ou nervosismo & $12,59(3,99-39,69)$ & $<0,001$ & $9,27(2,58-33,25)$ & $<0,001$ \\
\hline $\begin{array}{l}\text { Se sentir mal por esses problemas de urina } \\
\text { ou bexiga }\end{array}$ & $0,18(0,06-0,47)$ & $<0,001$ & $0,40(0,13-1,15)$ & 0,090 \\
\hline Uso de protetores higiênicos & $0,31(0,12-0,78)$ & 0,013 & $0,55(0,19-1,58)$ & 0,268 \\
\hline Preocupação em cheirar urina & $6,49(2,35-17,88)$ & $<0,001$ & $2,85(0,96-8,49)$ & 0,058 \\
\hline
\end{tabular}

IC, intervalo de confiança; IU, incontinência urinária; KHQ, King Health Questionnaire; RC, razão de Chance; RN, recém-nascido. Testes estatísticos: regressão logística univariada e regressão ajustada pela IU

\section{Discussão}

Na presente pesquisa, verificou-se que a maioria dos longevos que relataram que a urina ou bexiga atrapalhava a vida eram viúvos, com baixo nível de alfabetização e do sexo feminino. Um estudo em mulheres idosas com idade média de 74 anos incontinentes e moradoras de uma instituição de longa permanência, identificou caracteristicas semelhantes, sendo a maioria da amostra viúva e com baixo nível de escolaridade (9).

Em relação ao nivel de alfabetização, um estudo com idosos evidenciou que a maioria dos entrevistados possuia baixo nivel escolar, o que influenciava na compreensão de percepção de saúde, exposições a atores de risco, estilo de vida saudável e tratamentos adequados para as questões urinárias (15).

Quanto ao sexo, uma pesquisa buscando verificar a prevalência de IU mostrou que as mulheres são as mais acometidas com sintomas urinários (16), o que pode ser explicado devido a questões anatômicas, obstétricas e hormonais. A anatomia da pelve feminina apresenta uma uretra mais curta, uma bexiga menor, além de órgãos apoiados sobre a bexiga, causando uma maior compressão. Isso pode levar a uma maior propensão a desenvolver sintomas urinários, principalmente infecção urinária (17). Com relação aos nossos resultados, há maior número de mulheres em relação aos 
homens, apontando que há um maior crescente de mulheres à medida que a população envelhece, esse resultado vai ao de encontro com outro estudo publicado anteriormente também (1).

Uma revisão bibliográfica mostrou que problemas urinários, como a IU, aumentam com a idade. A explicação para isso, segundo os autores, se deve à diminuição da capacidade da bexiga em reter urina com o passar dos anos, diminuição do nivel de estrógeno, principalmente pós-menopáusica em mulheres, e aparecimento de doenças crônicas que podem aumentar os sintomas urinários (18).

O questionário utilizado para verificar a qualidade de vida foi originalmente elaborado pensando em mulheres com presença de incontinência urinária, tendo em vista que avalia a qualidade de vida relacionada a IU, sendo aplicado de forma independente ou respondida pelos próprios entrevistados. Em nosso estudo, esse questionário foi adaptado tanto para homens quando para mulheres com e sem presença de IU. Outra modificação foi em virtude dos avaliados serem longevos e, a maioria, com baixa escolaridade: optou-se pelo pesquisador ler as questões para os entrevistados, muitas vezes, exigindo modificações de palavras ou termos que os idosos não conheciam ou não entendiam para uma melhor compreensão da pergunta, essa mesma modificação já tinha sido previamente utilizada em outro estudo (13).

Quanto às limitações no desempenho de tarefas, a maior frequência de relato de limitação foi nas atividades fora de casa, o achado corrobora com um estudo que relata que as atividades fora de casa são as mais impactantes (19).

Quanto às limitações físico-social, a limitação ocorreu em maior frequência durante viagens, atividades físicas e visitar amigos e parentes. Um estudo demonstrou que a restrição social foi a queixa principal das mulheres que tinham IU, pois as impedia de sair de casa, ir a festas e clubes, fazer viagens longas e frequentar lugares. O mesmo aconteceu nas relações pessoas, houve limitação evidente com relação ao atrapalhar o companheiro e a relação sexual, já com relação ao incomodo de familiares, não houve nenhum relato disso. Nesse mesmo estudo supracitado, verificou que a vida sexual e com companheiro era prejudicada (18)

Nos itens de impacto da IU, foram relatados: ir muitas vezes ao banheiro, vontade forte de urinar e dificil controlar, vontade forte de urinar com presença de perda urinária, molhar a cama durante o sono, acordar muitas vezes para ir ao banheiro e infecção urinária. Foi possivel verificar que o grupo sem IU e incontinentes tinham os mesmos sintomas, ficando a ideia de que não é somente ter IU que atrapalha, pode ser frequência miccional, levantar à noite para ir ao banheiro, uso de diuréticos, ou não encontrar um banheiro de fácil acesso, como mostram pesquisas relacionadas à incontinência urinária que evidenciaram em seus estudos uma ligação direta entre pior qualidade de vida e a necessidade de ir muitas vezes ao banheiro, interromper o sono durante a noite pelo desejo de urinar $(20,21)$.

Quanto às emoções, se sentir mal pelos problemas de urina ou bexiga, sentimento de depressão e de ansiedade ou de nervosismo, foram sentimentos associadas com a bexiga ou urina atrapalhar a vida relatados pelos entrevistados com relação estatisticamente significativa. Esse estudo vai ao encontro do já publicado na literatura, que traz que a perda de urina gera sentimentos de depressão, levando ao isolamento social, baixa autoestima (17). Outro fator é o embaraço social, e a vergonha em procurar tratamento, se sentir desconfortável, podendo levar ao isolamento e a sintomas depressivos. A própria depressão poderia também contribuir para uma demora maior na busca por tratamento, tornando-se um círculo vicioso. Portanto, é importante considerar a mentalidade e a condição de humor ao gerenciar IU (22). Outra questão é que os sentimentos de ansiedade ativam o sistema parassimpático levando ao aparecimento de sintomas urinários como a bexiga hiperativa (23). O que se pode sugerir que a independência funcional e o comprometimento emocional estão diretamente relacionados.

Outro ponto importante é que a eliminação da urina contida na bexiga depende da interação do trato urinário com o sistema nervoso central (SNC) e do sistema nervoso periférico (SNP) (24), como 
o sistema nervoso está ligado com as emoções, sentimentos de ansiedade, depressão e outros podem inferir diretamente na IU (17).

Em relação ao sono e à energia, apesar de não apresentar relação estatisticamente significativa, houve um maior relatado de limitação em se sentir desgastado ou cansado e limitação durante o sono nos entrevistados que relataram que atrapalhava a vida. Em pesquisa realizada com 54 mulheres com IU, constatou-se que o aumento da frequência urinária noturna está relacionado à alteração do sono, diminuição da energia, e apresenta prejuizo emocional (14).

Já as medidas de gravidade, apresentaram limitação com associação estatisticamente significativa os longevos com desconforto urinário que relataram que precisavam usar protetores higiênicos, controlar a quantidade de líquido ingerida e se preocupavam em cheirar a urina. Esses achados vão ao encontro do publicado em outra pesquisa que evidenciou que grande parte dos indivíduos que possuem perda urinária diminui o consumo de líquidos ingeridos, principalmente água e principalmente no período da noite pelo medo de aumentar a frequência urinária e o volume de perda. Eles ainda relatam que o uso de protetores higiênicos, como um hábito frequente ao sair de casa, muitas vezes, causando incomodo e desconforto, aumentando a segurança e diminuindo a preocupação ao sair de casa e estar cheirando a urina, podendo levar ao aparecimento de infecção urinária (3).

Assim, o aumento das frequências urinarias diurnas e noturnas referidas foram os fatores que mais impactaram na QV. Esse aumento está diretamente relacionado com a limitação das atividades da vida diária, gerando alterações psicológicas, interferência no relacionamento pessoal e isolamento social, igualmente atrapalhando a qualidade do sono e a energia das mulheres incontinentes (8).

Os escores com maior impacto negativo na QV foram as medidas de gravidade com total de 77 pontos, limitação nas relações pessoais com 56 pontos e sono/energia com 37 pontos. Em uma pesquisa foi verificado também, que o domínio medidas de gravidade foi o mais afetado, indicando que o estilo de vida acaba sendo modificado devido às questões urinárias, uso proteção diariamente e ingestão menor de líquidos, além de constantemente apresentarem sentimentos como vergonha e constrangimento. Quanto à questão do sono, os autores também evidenciaram um alto escore, podendo ser explicado pela noctúria, já que os idosos podem acordar pelo desejo miccional e acabar perdendo o sono (25).

Como limitação do estudo, foi percebida a dificuldade de compreensão por parte dos longevos de algumas perguntas do questionário $\mathrm{KHQ}$, não ter aplicado o questionário prévio para avaliar a cognição, além de ficar claro e evidente o sentimento de vergonha e constrangimento em falar sobre um tema tão intimo. Em alguns casos foi percebida uma omissão dos reais sintomas pelos mesmos motivos. Além de poucos estudos envolvendo longevos para que pudessem ser discutidos os resultados com pesquisas com idosos da mesma idade, pois se tratando de saúde vesical a idade é fator extremamente decisivo.

Este estudo identificou uma relação estatisticamente significativa entre o relato do funcionamento do sistema urinário atrapalhar a vida de modo geral com a análise do questionário Kings Health Questionnaire (KHQ) em longevos (85 anos ou mais), mesmo sem relatar perda urinária. 0 impacto da perda urinária sobre a qualidade de vida mensurado pelo $\mathrm{KHQ}$ foi relacionado com a queixa de o funcionamento do sistema urinário atrapalhar a vida dos longevos e a perda urinária foi um fator de confusão para alguns dos domínios relacionados. Nos modelos finais de análise estatistica, permaneceram relacionadas à queixa de "a bexiga ou urina atrapalhar a vida" as situações em que os participantes relacionavam comprometimentos emocionais e funcionais relacionados à independência fora do domicílio com alterações na funcionalidade do sistema urinário (limitação de tarefas fora de casa; limitação em viagens; vontade forte de urinar; e dificil de controlar; sentimento de depressão; ansiedade ou nervosismo)

Como o percentual de continentes e de incontinentes do grupo que relatava não atrapalhar a 
vida foi muito próximo, a melhora da qualidade de vida em longevos através da abordagem terapêutica do funcionamento do sistema urinário parece precisar perpassar de somente questões sobre a presença de perda urinária, mas fazer a identificação de outras questões como a frequência mictória e a sua rotina no cotidiano dos longevos. Esses dados reforçam a importância do diário miccional e de investigar quais mecanismos estão associados a piora da qualidade de vida pelo comprometimento da função vesical. Além disso, atentam para uma abordagem multiprofissional, para o cuidado das questões emocionais e buscar a prevenção e/ou retorno para a rotina de tarefas fora do ambiente domiciliar.

\section{NOTAS}

Parte deste trabalho é resultado de dissertação de mestrado em Gerontologia Biomédica da Escola de Medicina da PUCRS de uma das autoras (LBJ), intitulada "Fatores relacionados ao desconforto urinário em longevos e longevas"

\section{Apoio financeiro}

O presente trabalho foi realizado com apoio da Coordenação de Aperfeiçoamento de Pessoal Nivel Superior - Brasil (CAPES) - Código de Financiamento 001

\section{Declaração de conflito de interesses}

Os autores declaram não haver conflitos de interesses relevantes ao conteúdo deste estudo.

\section{Contribuições dos autores}

Todos os autores fizeram contribuições substanciais para concepção, ou delineamento, ou aquisição, ou análise ou interpretação de dados; e redação do trabalho ou revisão crítica; e aprovação final da versão para publicação.

\section{Disponibilidade dos dados e}

\section{responsabilidade pelos resultados}

Todos os autores declaram ter tido total acesso aos dados obtidos e assumem completa responsabilidade pela integridade destes resultados.

\section{Referências}

1. IBGE. Instituto Brasileiro de Geografia e Estatística. Censo de 2010. 2010. Disponivel em: http://www.ibge. gov.br/home/estatistica/populacao/censo2010/default.shtm. Acesso em: 12 mar. 2017.

2. Silva SPZ, Marin MJS, Rodrigues MR. Condições de vida e de saúde de idosos acima de 80 anos. Rev Gaúcha Enferm. 2015:36(3):42-8. https://doi.org/10.1590/19831447.2015 .03 .50263

3. Honório MO, Santos SMA. Incontinência urinária e envelhecimento: impacto no cotidiano e na qualidade de vida. Rev bras enferm. 2009;62(1):51-6. https://doi. org/10.1590/S0034-71672009000100008

4. Lopes MHB, Higa R. Restrições causadas pela incontinência urinária à vida da mulher urinária à vida da mulher. Rev esc enferm USP. 2006;40(1):34-41. https:// doi.org/10.1590/S0080-62342006000100005

5. Bomfim IQM, Soutinho SRS, Araújo SRS. Comparação da qualidade de vida das mulheres com incontinência urinária atendidas no sistema de saúde público e privado. Revista cientifica ciências biológicas e da saúde. 2014; 16(1):19-24.

6. Flores MN, Santos NC, Féra P, Glashan RQ. Incontinência urinária em idosas institucionalizadas. Sinopse de Urologia 2004;8(3):70-4

7. Jrez-roig J, Souza DLB, Lima KC. Incontinência urinária em idosos institucionalizados no Brasil: uma revisão integrativa. Rev bras geriatr gerontol. 2013;16(4):865-79. https://doi.org/10.1590/S1809-98232013000400020

8. Morsch P, Pereira GN, Navarro JHN, Navarro JHN, Trevisan MD, Lopes DGC, Bós AJG. Clinical characteristics and social determinants in a sample of non-homebound elderly. Cad Saude Publica. 2015:31(5):1025-34. https:// doi.org/10.1590/01021-311X00053014

9. Soliman Y, Meyer R, Baum N. Falls in the elderly secondary to urinary symptoms. Rev Urol. 2016;18(1):28-32.

10. John G, Bardini C, Combescure C, Dällenbach $P$. Urinary incontinence as a predictor of death: a systematic review and meta-analysis. PLoS One. 2016;11(7):e0158992. https://doi.org/10.1371/journal. pone.0158992

11. Rigo II, Bós AJG. Disfunção familiar em nonagenários e centenários, importância das condições de saúde e suporte social. Cien Saude Colet [Internet]. 2019 [cited 2020 Mar 26]; Available from: http://www.cienciaesaudecoletiva.com.br/artigos/disfuncao-familiar-em-nonagenarios-e-centenarios-importancia-das-condicoes-de-saude-e-suporte-social/17390?id=17390

12. Brasil. Ministério da Saúde. Secretaria de Atenção à Saúde, Departamento de Atenção Básica. Cadernos de atenção básica, n. 19. Envelhecimento e saúde da pessoa idosa. 1st ed. Brasilia, DF: Ministério da Saúde; 2006 
13. Zanella AK. Avaliação da consciência da musculatura do assoalho pélvico e sua relação com a incontinência urinaria em idosas, [tese]. Porto Alegre (RS): Pontificia Universidade Católica do Rio Grande do Sul; 2018.

14. Fonseca ESM, Camargo ALM, Castro RA, Sartori MG, Fonseca MCM, Lima GR, Girão MJBC. Validação do questionário de qualidade de vida (King's Health Questionnaire) em mulheres brasileiras com incontinência urinária. Rev Bras Ginecol Obstet. 2005; 27(5):235-42. https://doi.org/10.1590/S0100-72032005000500002

15. Berlezi EM, Dal Bem A, Leite CAMT, Bertolo EM. Incontinência urinária em mulheres no período pósmenopausa: um problema de saúde pública. Rev. Bras. Geriatr. Gerontol, 2009; 12(2):159-73. https://doi. org/10.1590/1809-9823.2009120200

16. Silva APM, Santos VLCG. Prevalência da incontinência urinária em adultos e idosos hospitalizados. Rev esc enferm USP. 2005:39(1): 36-45. https://doi.org/10.1590/ S0080-62342005000100005

17. Neto ONV. Infecção do trato urinário. Medicina (Ribeirão Preto). 2003:36(1):365-9. https://doi.org/10.11606/ issn.2176-7262.v36i2/4p365-369

18. Higa R, Lopes MHBM, Reis MJ. Fatores de risco para incontinência urinária na mulher. Rev esc enferm USP 2008;42(1):187-92. https://doi.org/10.1590/S0080$\underline{62342008000100025}$

19. Miner, JRPB. Economic and personal impact offecal and urinary incontinece. Gastroenterology. 2004:126:S8S13. https://doi.org/10.1053/j.gastro.2003.10.056

20. Oliveira JM, Salgado LBG, Schmitt SCB, Rosa LCL. Correlação entre sintomas urinários e qualidade de vida em mulheres com incontinência urinária. Fisioterapia e Pesquisa 2007:14(3):12-7

21. Sampselle CM 1, Harlow SD, Skurnick J, Brubaker G, Bondarenko EU. Preditores de incontinência urinária e impacto na vida em mulheres etnicamente diversas na perimenopausa. Obstet Gynecol.2002; 100(6):1230-8. https://doi.org/10.1097/00006250-200212000-00013

22. Heilberg IP, Schor, N. Abordagem diagnóstica e terapêutica na infecção do trato urinário: ITU. Rev. Assoc. Med. Bras. 2003 Jan;49(1):109-16. https://doi. org/10.1590/S0104-42302003000100043

23. Bontempo APS,Alves AT,Martins GS, Jácomo RH,Malschik DC, Menezes RL. Fatores associados à síndrome da bexiga hiperativa em idosas: um estudo transversal. Rev Bras Geriatr Gerontol., 2017; 20(4): 474-83

24. Marinho, AR; Leal, BB; Flister, JS; Bernardes NO; Rett, MT. Incontinência urinária feminina e fatores de risco. Fisioterapia Brasil, 2006;7(4):301-6. https://doi. org/10.33233/fb.v7i4.1921

25. Faria CL, Moraes JR, Monnerat BRD, Veridiano KA, Hawerroth PAMM, Fonseca SC. Impacto do tipo de incontinência urinária sobre a qualidade de vida de usuárias do Sistema Único de Saúde no Sudeste do Brasil. Rev Bras Ginecol Obstet. 2015:37(8):374-80. https://doi.org/10.1590/SO100-720320150005394

\section{Luisa Braga Jorge}

Fisioterapeuta, pós-graduada em Fisioterapia Pélvica pela INSPIRAR (Curitiba, PR, Brasil). Mestre e doutoranda em Gerontologia Biomédica pela Pontifícia Universidade Católica do Rio Grande do Sul (PUCRS, Porto Alegre, RS, Brasil).

\section{Josemara de Paulo Rocha}

Fisioterapeuta, Mestre em Envelhecimento Humano, Especialista em Saúde do Idoso pela Universidade de Passo Fundo (UPF, Passo Fundo, RS, Brasil). Doutoranda do Programa de Pós-graduação em Gerontologia Biomédica da Escola de Medicina da Pontifícia Universidade Católica do Rio Grande do Sul (PUCRS, Porto Alegre, RS, Brasil).

\section{Ângelo José Gonçalves Bós}

Médico. Especialista em Geriatria e Gerontologia pelo IGG da Pontifícia Universidade Católica do Rio Grande do Sul (PUCRS, Porto Alegre, RS, Brasil) e Saúde Comunitária pela Unidade Sanitária Murialdo. Doutor em Saúde Comunitária pela Tokai University (Japão). Pós-doutorado em Epidemiologia do Envelhecimento pelo Instituto Nacional Americano sobre o Envelhecimento e Escola de Saúde Pública da Universidade de Johns Hopkins (Baltimore, EUA). Pós-doutorado no Departamento de Saúde e Participação Social do Tokyo Metropolitan Institute of Gerontology (Japão). Professor permanente do Programa de Mestrado e Doutorado em Gerontologia Biomédica e Professor Titular da Escola de Medicina da Pontifícia Universidade Católica do Rio Grande do Sul (PUCRS, Porto Alegre, RS, Brasil).

\section{Rodolfo Herberto Schneider}

Médico, especialista em Clínica Médica e Geriatria; Mestre e Doutor em Ciências da Saúde pela Pontifícia Universidade Católica do Rio Grande do Sul (PUCRS, Porto Alegre, RS, Brasil); Professor Permanente do Programa de Pós-Graduação em Gerontologia Biomédica do Instituto de Geriatria e Gerontologia da PUCRS. Membro da Comissão Coordenadora do Programa de Pós-Graduação em Gerontologia Biomédica. Coordenador da Comissão Cientíica do Instituto de Geriatria e Gerontologia. Consultor da CAPES na Área Interdisciplinar. Chefe do Serviço de Densitometria Óssea do Hospital São Lucas da PUCRS, Pesquisador responsável pelo Grupo de Pesquisa em Envelhecimento Osteomuscular e Osteoporose (GEOMO) - CNPQ (PUCRS, Porto Alegre, RS, Brasil).

\section{Endereço para correspondência}

Luisa Braga Jorge

Rua Senador Salgado Filho 355, apto. 701

São Bento, 95700000

Bento Gonçalves, RS, Brasil 